\title{
A inserção do psicólogo na residência multiprofissional em saúde: um relato de experiência em oncologia
}

The inclusion of psychologists in multidisciplinary home health: a report of experience in oncology

La inserción del psicólogo en la residencia multiprofesional en salud: un relato de experiencia en oncología

\author{
Jamile Luz Morais* \\ Emily Suelen Antunes de Castro ${ }^{* *}$ \\ Airle Miranda de Souza**
}

\begin{abstract}
Resumo
Este trabalho tem como objetivo relatar a experiência de duas psicólogas e sua inserção no programa da Residência Multiprofissional em Saúde na área de Oncologia do Hospital Universitário João de Barros Barreto. Pioneira no Estado do Pará, a Residência Multiprofissional, baseando-se nos princípios da universalidade, integralidade e equidade, disponibiliza à população um atendimento integral à saúde por meio da atuação direta de profissionais de Psicologia, Nutrição, Fisioterapia, Biomedicina, Enfermagem, Farmácia, Terapia Ocupacional e Odontologia, nos três níveis de atenção à saúde. Nessa experiência salientamos a importância do psicólogo inserido em equipe multiprofissional de saúde, apontando para a especificidade do seu papel com o paciente e com essa equipe. Por fim, destacamos a oportunidade de compartilhar percepções, sentimentos e condutas clínicas nos grupos desenvolvidos no ambiente hospitalar, centrados na tarefa de abordar o processo de morte e o morrer.
\end{abstract}

Palavras-chave: Psicologia. Residência multiprofissional. Oncologia.

\footnotetext{
Doutoranda do Programa de Pós-graduação em Psicologia Social na Pontifícia Universidade Católica de São Paulo (PUCSP), mestra em Psicologia, especialista em Oncologia na Modalidade Residência Multiprofissional em Saúde pelo Hospital Universitário João de Barros Barreto (HUJBB), em parceria com a Universidade Federal do Pará (UFPA), psicóloga.

** Especialista em Oncologia na Modalidade Residência Multiprofissional em Saúde pelo Hospital Universitário João de Barros Barreto (HUJBB), em parceria com a Universidade Federal do Pará (UFPA), atua na Clínica de Hematologia do Hospital Ophir Loyola, psicóloga.

*** Doutora em Ciências Médicas pela Universidade Estadual de Campinas (Unicamp), professora da Faculdade e do Programa de Pós-graduação em Psicologia na Universidade Federal do Pará (UFPA), tutora de Psicologia do Programa de Residência Multiprofissional em Saúde do Hospital Universitário João de Barros Barreto (HUJBB), onde também coordena o Laboratório de Estudos do Luto e Saúde (LAELS), psicóloga.
} 


\begin{abstract}
This paper aims to report the experience of two psychologists and their insertion in the Multidisciplinary Residency Program in Health in Oncology, University Hospital João de Barros Barreto. A pioneer in the State of Pará, the Multidisciplinary Residency, based on the principles of universality, comprehensiveness, and equity, the population provides a comprehensive healthcare through the direct intervention of psychologists, nutrition, physiotherapy, bio-medicine, nursing, pharmacy, therapy occupational and dentistry, the three levels of health care. In this experiment to emphasize the importance of the psychologist inserted in multi-professional health team, pointing to the specificity of its role with the patient and with this team. Finally, we highlight the opportunity to share perceptions, feelings and conduct in clinical groups developed in the hospital environment focused on the task of approaching the process of death and dying.
\end{abstract}

Keywords: Psychology. Multiprofessional residence. Oncology.

\title{
Resumen
}

El objetivo de este trabajo es relatar la experiencia de dos psicólogas y su inserción en el Programa de Residencia Multiprofesional en Salud, en el área de Oncología del Hospital Universitario João de Barros Barreto. El Programa de Residencia Multiprofesional, es pionero en el Estado de Pará - Brasil, y basándose en los principios de la universalidad, integralidad e equidad, ofrece a la población una atención integral en lo que se refiere a la salud, a través de la intervención directa de profesionales de psicología, nutrición, fisioterapia, biomedicina, enfermería, farmacia, terapia ocupacional y odontología, en los tres niveles de atención a la salud. En esta experiencia resaltamos la importancia del psicólogo inserido en un equipo multiprofesional de salud, destacando la especificidad de su papel en relación al paciente y al equipo. Y para terminar, destacamos la oportunidad de compartir percepciones, sentimientos y conductas clínicas en los grupos desarrollados en el ambiente del hospital, concentrados en la tarea de abordar el proceso de muerte y el morir.

Palabras clave: Psicología. Residencia multiprofesional. Oncología.

\section{Introdução}

residência multiprofissional em saúde é um programa de pós-graduação
em nível lato sensu. Pioneira no Estado do Pará, é desenvolvida por
meio do Hospital Universitário João de Barros Barreto (HUJBB), 
em parceria com outras instituições de saúde. ${ }^{1}$ Vinculado à Universidade Federal do Pará (UFPA), o programa garante uma certificação em nível de especialização, destacando-se entre as suas especificidades: duração de 24 meses, dedicação exclusiva, carga horária de 5760 horas, sendo 1160 horas de atividades teóricas ou teórico-práticas e 4600 horas de atividades práticas, distribuídas em 60 horas semanais, baseado em um regime educativo em serviço (Brasil, 2006).

No que tange à sua origem, consta em Feuerweker (2002 citado por Silva, 2010) que, como modalidade de formação, as residências em saúde existem no Brasil desde a década de 1940. Na década posterior, houve um crescente aumento e a consequente consolidação dessa modalidade na área médica, instituindo-se como uma alternativa de pós-graduação restrita nessa área. Esse fato contribuiu para a posse do termo "residência" por parte da Medicina, salientando que, embora várias profissões se utilizem dessa expressão para apontar o conjunto de saberes e poderes, existe uma apropriação desse substantivo pela categoria médica, a partir do decreto 80.281/1977 e da Lei 6.932/1981, que definem a residência médica como uma modalidade de ensino destinada a médicos (Feuerweker, 1998, citado por Dallegrave, 2008).

Entretanto, anos se passaram e, com a implantação do Sistema Único de Saúde (SUS), surgiu, em 1988, a necessidade de se atribuir importância ao ideal da integralidade da atenção. Assim, na $8^{\text {a }}$ Conferência Nacional de Saúde (CNS), tida como um marco histórico com relação à saúde no Brasil, o paradigma de saúde foi reformulado. Nesse panorama, a saúde deixou de ser vista apenas como ausência de doença para ser concebida como decorrência das condições de vida e trabalho. Tal concepção levou ao acesso igualitário de todos aos serviços de promoção, proteção e recuperação da saúde, colocando como uma das questôes fundamentais a atenção integral à saúde e a participação social (Brasil, 2006).

Posteriormente, na $10^{\text {a }} \mathrm{CNS}$, reafirmou-se a necessidade de consolidar o SUS, com todos os seus princípios e objetivos, atentando à importância da ação interdisciplinar no âmbito da saúde e o reconhecimento imprescindível das ações realizadas pelos diferentes profissionais de nível superior, marcando um avanço no que diz respeito à saúde, especialmente com relação à hegemonia do modelo médico-hegemônico existente até então.

Como fruto desse contexto sóciopolítico, a RMS foi regulamentada no ano de 2005, pela Lei 11.129, sancionada pelo Presidente da República no

Como Hospital Público Ophir Loyola (HOL), o Hospital Universitário Betina Ferro de Sousa (HBFS) e unidades básicas de saúde. 
dia 30 de junho, o qual implantou, no âmbito do Ministério da Educação, a Comissão Nacional de Residência Multiprofissional em Saúde (Brasil, 2006).

Em Belém, no HUJBB, instituiu-se a residência multiprofissional em saúde no ano de 2010, sendo ofertadas 20 vagas, assim distribuídas: 10 para a área de concentração em Oncologia (Psicologia, Biomedicina, Nutrição, Farmácia, Enfermagem e Odontologia) e 8 vagas para Saúde do Idoso (Psicologia, Farmácia, Enfermagem e Fisioterapia). No ano seguinte, o número de vagas foi ampliado para 28 profissionais, passando a fazer parte do programa terapeutas ocupacionais, assistentes sociais e nutricionistas em Saúde do Idoso e fisioterapeutas em Oncologia.

O ingresso dos profissionais ocorre por meio das seguintes etapas: prova objetiva eliminatória (contendo 50 questôes divididas entre conhecimentos específicos e um eixo comum às especialidades), avaliação curricular e entrevista. No que se refere à demanda, observou-se, no processo seletivo de 2011, um aumento significativo de inscritos na seleção do ano anterior, totalizando 350 participantes.

Quando inserido no programa, o profissional, subsidiado pelo treinamento em serviço mediante seu preceptor de campo e pelo empreendimento teórico por meio do tutor, desenvolve trabalho em sua especificidade, visando à atuação interdisciplinar. Dessa maneira, o paciente, seja oncológico ou idoso, é assistido por todas as formações acadêmicas, as quais procuram se entrecruzar no intuito de compreender, da melhor forma possível, partindo de um olhar biopsicossocial, ambas as clientelas. Dessa forma, verifica-se que a residência multiprofissional constitui-se não só como uma opção de qualificação profissional, uma especialização, mas também como uma oportunidade de exercer os conhecimentos adquiridos durante a graduação em conjunto com outras formaçōes.

No que diz respeito à origem do Programa, este se fundamentou no próprio conceito de saúde, concebido pela $8^{\text {a }}$ Conferência Nacional de Saúde (CNS), na qual se estabeleceu que esta seria um "direito de todos e dever do Estado". Esse pressuposto ampliou a compreensão da relação saúde-doença como decorrência das condições de vida e trabalho, bem como do acesso igualitário de todos aos serviços de promoção, proteção e recuperação da saúde, colocando como entre as questôes fundamentais a integralidade da atenção à saúde e a participação social (Brasil, 2006).

Nessa perspectiva, o Ministério da Saúde (MS), acreditando tanto na 
formação de profissionais quanto na possibilidade de mudança do desenho tecnoassistencial do SUS, vem apoiando as residências multiprofissionais desde 2002, por meio do projeto ReforSUS. ${ }^{2}$ Aliás, é justamente a intrínseca característica da interdisciplinaridade que confere caráter inovador aos programas de residência multiprofissional. Nesse contexto, foram incluídas 14 categorias profissionais da saúde, dentre as quais estão assistentes sociais, psicólogos, biólogos, biomédicos, profissionais de Educação Física, enfermeiros, farmacêuticos, fisioterapeutas, fonoaudiólogos, médicos, médicos veterinários, nutricionistas, odontólogos e terapeutas ocupacionais (Brasil, 2006).

Portanto as diferentes profissões, trabalhando em conjunto, visam a uma formação intercategorias, como bem afirma Zannon (1994), ao conceituar interdisciplinaridade:

Ações conjuntas, integradas e inter-relacionadas, de profissionais de diferentes procedências quanto à área básica do conhecimento. Implica a tomada de decisão acerca das condutas profissionais, levando em conta os aspectos relativos às diversas disciplinas, vale dizer, as diversas dimensões da vida humana, de seu estudo e da intervenção profissional (Zannon, 1994, p. 16).

Esse modo de operar tem por finalidade a formação coletiva inserida no mesmo campo de trabalho, sem, contudo, deixar de priorizar e respeitar os núcleos específicos de saberes de cada profissão. No caso da oncologia, os profissionais procuram atuar nos três níveis de atenção à saúde, considerando, além da recuperação e reabilitação do paciente, a educação em saúde como forma de promoção e prevenção.

Entretanto, ao mesmo tempo em que se reconhece a importância do trabalho interdisciplinar, verifica-se a necessidade de explicitar o trabalho do psicólogo no contexto hospitalar, ambiente apontado por Ismael (2005) como um lócus de atuação relativamente novo. Em razão disso, muitas são as expectativas que permeiam a tarefa do psicólogo no hospital, as quais comumente não refletem a realidade desse profissional. Um estudo realizado por Moré, Crepaldi, Queiroz, Wendt \& Cardoso (2004) demonstra o desconhecimento com relação não só à Psicologia hospitalar, mas com a própria ciência psicológica, a qual muitas vezes acaba sendo reduzida ao modelo clínico.

Projeto de financiamento para impulsionar a implantação dos polos de capacitação, formação e educação permanente de recursos humanos em saúde. 
Diante do exposto, este artigo propõe relatar a experiência de ingresso de duas psicólogas no Programa de Residência Multiprofissional em Oncologia no Hospital Universitário João de Barros Barreto, discutindo teoricamente e apresentando sua função dentro da equipe multiprofissional e da instituição de saúde.

\section{Revendo caminhos: a inserção no programa e o papel do cuida(dor) psicólogo}

Ingressamos no programa no final do ano de 2010, integrando a primeira turma da Região Norte do Brasil formada por intermédio dessa modalidade de aperfeiçoamento profissional. Até então, o que conhecíamos sobre a residência era aquela destinada à formação médica, incluindo a escuta dessas experiências nas falas de amigos ou de outros colegas da área. $\mathrm{O}$ que seria então ser residente inserido em um programa multiprofissional? As expectativas e um grande entusiasmo em iniciar o trabalho nos contagiavam, assim como a certeza de que o caminho merecia ser trilhado e construído. Também estávamos certas de que nosso trabalho seria muito importante no desenvolvimento e na abertura de espaços que viriam posteriormente.

Outras questôes foram suscitadas acerca de como esta poderia se realizar: como pode existir uma residência não direcionada à formação médica? Como pode ser possível profissionais de diversas especialidades formaremse com base em um eixo comum, em nosso caso, a oncologia?

Ao mesmo tempo, embora atravessadas por essas interrogaçóes, percebíamos na residência multiprofissional um caminho não só para nossa qualificação profissional específica em psicologia da saúde e oncologia, mas também como excelente oportunidade de desenvolver habilidades de se trabalhar em equipe, de trocar conhecimentos e aprender com a experiência de outros profissionais que não psicólogos.

Imbuídas de tais expectativas, submetemo-nos ao processo de seleção para concorrer a duas vagas ofertadas para Psicologia. Nessa especialidade, 20 profissionais inscreveram-se, passando para a fase final (entrevista e análise curricular) 6 candidatos, segundo as normas do edital de seleção 2010, conforme o qual seriam aprovados para a etapa final os 6 primeiros colocados na prova escrita, que correspondem a três vezes o número de vagas disponíveis.

Submetemo-nos, então, à entrevista final em busca de uma experiência única: pôr em prática nossos conhecimentos específicos integrados a 
outros saberes, intervindo em uma equipe multiprofissional. Cabia a nós caminhar e construir o ser residente psicólogo.

Uma vez aprovadas no processo de seleção, começamos a atuar, juntamente com as outras categorias profissionais, nos três níveis de atenção à saúde, a saber: na promoção, prevenção, tratamento e reabilitação do paciente oncológico. Intercalando com os níveis de atenção à saúde, o programa iniciou e terminou suas atividades no terceiro nível da atenção, no qual conhecemos a rotina hospitalar e as principais clínicas onde são internados pacientes com neoplasias diversas.

Atuamos aproximadamente dois meses em cada clínica especializada, como a clínica de pneumologia, hematologia, ginecologia e mastologia, urologia, cirúrgica, pediatria, cabeça e pescoço, e cuidados paliativos e oncológicos. Em cada uma delas, sob supervisão do preceptor de campo e tutor, realizamos o acompanhamento psicológico do paciente desde o início da internação até a alta hospitalar, por meio tanto da busca ativa quanto da interconsulta, nos casos em que o profissional é solicitado pela equipe de saúde a prestar assistência. Tem-se que o enfoque do psicólogo são os aspectos psicológicos do adoecer, os quais são manifestados, segundo Simonetti (2004), por intermédio dos sentimentos, da fala, dos pensamentos, dos comportamentos, das atitudes, das fantasias, das lembranças, dos sonhos, dos conflitos, do estilo de vida e do estilo de adoecer.

Trabalhamos de modo a destinar ao paciente uma escuta capaz de oferecer o manejo de seu sofrimento em decorrência da doença e hospitalização, possibilitando a emergência do sujeito como um todo, para além de sua patologia, considerando sua particularidade com base em uma visão integral.

Por outro lado, ressaltamos que nossa tarefa no hospital não se restringiu somente ao atendimento direcionado ao paciente, pois se estendeu aos familiares e à equipe de saúde. Intervimos no intuito de proporcionar um olhar diferenciado em torno do paciente, o qual, muitas vezes, por estar doente, perde sua autonomia, despersonalizando-se em meio à institucionalização promovida pela internação hospitalar.

Quando se trata do paciente oncológico, a importância do cuidar do paciente e dos familiares se intensifica, considerando as representações sociais que o câncer traz consigo, pois, como nos pontua Leshan (1994, p. 19): "O câncer não é apenas uma doença; são diversas doenças relacionadas que afetam, de várias maneiras, diferentes partes do corpo humano. E, 
em parte por este motivo, continua sendo a mais misteriosa das doenças graves". É fato que, apesar de todos os avanços no tratamento, as causas do câncer continuam sendo motivos de controvérsias. Sontag (2004), ao tocar nas doenças não totalmente compreendidas pela Medicina, remetese ao câncer, dizendo:

Assim como hoje, embora todos reconheçam que o câncer é um enigma não resolvido, admite-se também de forma geral que o câncer tem múltiplas causas [...] A ideia de que uma doença pode ser explicada apenas por uma variedade de causas é exatamente a característica da reflexão acerca de doenças, cuja causa não é compreendida (Sontag, 2004, p. 55).

O câncer é um inimigo a ser combatido, que deve ser retirado, "jogado fora" do organismo. Por esse motivo é que desde o diagnóstico paciente e familiar já requerem cuidados. Para Sontag (2004), receber um diagnóstico de câncer iguala-se a receber uma sentença de morte, sendo comumente visto como uma punição e acompanhado da pergunta: "Porque comigo?", "Por com ele (a)?". Assim, o diagnóstico de câncer coloca paciente e familiar diante da própria finitude, confrontando o ser humano com aquilo que ele nega e não pode simbolizar: a morte.

De acordo com Liberato \& Carvalho (2008), receber um diagnóstico de uma doença grave, como o câncer, e submeter-se aos seus tratamentos habituais, por si só, ocasionam elementos estressantes, entre os quais estão a dor e a ansiedade quanto ao corpo mutilado e à morte. Em estudo realizado por Green e Green (1992) citado por Bromberg (1998), 70\% dos pacientes com câncer se queixam de dor, a qual está diretamente associada com o sofrimento pela proximidade da morte, o que exige apoio físico, psicológico, espiritual e social, isto é, de uma equipe multiprofissional.

Também não podemos desconsiderar a dor dos familiares, a qual, por ser de cunho subjetivo, muitas vezes é negligenciada pela equipe de saúde. Ao tratar da participação da família no processo de adoecimento do paciente oncológico, Bromberg (1998) afirma que todos os cuidados relativos à família serão inválidos se esta não for considerada "a partir de uma experiência de perda que não terá início apenas no momento de morte" (p. 197), mas antes mesmo do diagnóstico. Salienta Franco (2008, p. 358): "Pode-se dizer que a crise decorrente da doença inicia-se antes do diagnóstico, quando a família tem alguma percepção ou interpreta sintomas como de risco ou une-se (ou fragmenta-se, pela dificuldade) para lidar com os sintomas e sistemas médicos". 
A autora afirma ainda que o modo pelo qual pacientes e familiares convivem com a fase pré-diagnóstica comumente sugerem os padrões que persistirão no momento de enfrentamento da realidade colocada. No diagnóstico, o que se destaca é a dificuldade de comunicação existente na tríade paciente-família-equipe de saúde, pois "algumas pessoas podem não querer compartilhar a notícia do diagnóstico com seus familiares (todos ou alguns) ou estes podem querer manter o paciente na ignorância" (Franco, 2008, p. 358).

Ao receber o diagnóstico, a família passa por toda uma reconfiguração em seu sistema, uma vez que este interfere nos projetos construídos por ela, os quais deverão ser mudados ou adiados. Instaura-se claramente uma crise familiar permeada de incerteza e ansiedade que atingirá tanto o paciente quanto sua rede de suporte. Nessa etapa, ambos vivem o luto antecipatório, definido por Kovács (1992) como:

O luto antecipatório é o processo que ocorre antes da morte concreta, devendo haver um trabalho com pacientes e familiares concernente a essa etapa. No caso do paciente, envolve perdas de si, da saúde, da vida e a separação das pessoas queridas. O luto antecipatório para os familiares envolve as perdas relacionadas ao adoecimento, a perspectiva de morte e a sensação de sobrecarga por parte dos cuidadores (Kovács, 1992, p. 394).

Diante disso, o trabalho com a família deve ser parte integrante da atuação do psicólogo, haja vista que dispensar cuidados simplesmente ao paciente não é suficiente para seu equilíbrio emocional. Ao cuidar da família, estamos cuidando automaticamente da saúde do paciente. Para Angerami-Camon (2002), os familiares representam um papel fundamental no tratamento do paciente, interferindo inclusive em seu estado de saúde, decidindo possíveis rejeições ou enfrentamentos com relação ao tratamento e efeitos colaterais.

Entretanto nada do exposto faz sentido se não considerarmos a atitude do psicólogo ante a morte, tendo em vista que, ao optar trabalhar na área de oncologia, esse profissional automaticamente escolhe conviver diariamente com a emergência de morte. Por isso tecer elaboraçóes teóricas a respeito da morte é uma tarefa necessária para quem trabalha com oncologia, porque é discutindo e lembrando que ela é a certeza da vida é que podemos vislumbrar dispositivos clínicos que subsidiem a escuta e o acompanhamento psicológico nos hospitais. 


\section{O psicólogo diante a emergência de morte}

De acordo com Kovács (1992), embora a morte seja um fenômeno natural da vida, o homem, pela dificuldade de dar sentido a ela, tende a negá-la, adiála, escamoteá-la, por não querer deparar com sua própria mortalidade. Freud (1996) assinala o fenômeno da morte como algo estranho a nós mesmos:

É verdade que a afirmação "todos os homens são mortais" é
mostrada nos manuais de lógica como exemplo de uma proposição
geral; mas nenhum ser humano realmente a compreende, e o
nosso inconsciente tem tão pouco uso hoje, como sempre teve,
para a ideia da sua própria mortalidade (Freud, 1996, p. 259).

Sob outra ótica, nossa impotência com relação à morte nos leva à necessidade de dar algum sentido a ela, simbolizá-la. Para a psicanálise, podemos apenas bordejar a morte, isto é, falar dela bem como encontrar rituais que possam dar algum significado à finitude. No que diz respeito ao paciente oncológico, podemos dizer que ele está a todo tempo confrontado com a angústia de morte, precisando, por isso, falar dela para, quem sabe, ser capaz de bordejála.

O câncer, definido como um conjunto de patologias provocadas pelo desenvolvimento de alterações em processo de divisão celular, segundo dados do Instituto Nacional do Câncer, o INCA, é tido na atualidade como um problema de saúde pública, sendo a segunda principal causa geral de morte por doença no mundo, responsável por 6 milhōes de óbitos anuais (INCA, 1997). Nesse ambiente, onde a emergência de morte se faz imperativa, há de se perguntar: como é que fica a atuação do psicólogo com esses pacientes, em especial aqueles internados para tratamento quimioterápico? Que direção dar ao tratamento?

Acredita-se que o papel do psicólogo consiste em sustentar a escuta de um sofrimento que não necessariamente pode ser significado, ainda mais quando se trata, no caso da leucemia, da impossibilidade de retirada do tumor. Essa situação tende a impedir o paciente de (re)significar a condição pela qual foi tomado. O resultado de tudo isso é que o sujeito, ao não encontrar palavras para dizer como se sente, cala-se diante da sua dor. Logo, o psicólogo acaba assumindo a função de testemunhar junto ao paciente a sua dor, escutando o silêncio, respeitando o tempo subjetivo deste e se colocando numa posição que leve o sujeito a sair desse ciclo e consiga, de algum modo, ao menos rodear aquilo que não tem explicação e nunca terá: a certeza da morte.

Faz-se necessário também um olhar cuidadoso àqueles que acolhem a dor, aos cuida(dores), sejam eles psicólogos, médicos, enfermeiros ou 
fisioterapeutas, destinados, muitas vezes, a viverem com o paciente a última etapa do ciclo de vida: o morrer. A morte do outro também sinaliza a morte de todo ser vivo, podendo favorecer angústias existenciais ou reabrir feridas relacionadas às perdas significativas ao longo do ciclo da vida. $\mathrm{O}$ agravo do estado de saúde ou o óbito daquele a quem a equipe investe cuidados pode mobilizar nos cuidadores culpa e sensação de fracasso, favorecendo, por sua vez, outros questionamentos. O que dizer aos familiares sobre a criança em processo de morte? Como comunicar que, para manter a vida, é necessário amputar um membro em que desse depende o sustento para viver? Com quem compartilhar os gritos dos sobreviventes diante da morte da pessoa amada, espalhados nas enfermarias, que marcam os que se ocupam em acolher dores?

A esse respeito, destacamos nossa participação nas atividades do Laboratório de Estudos do Luto e Saúde (Laels), projeto de pesquisa e extensão desenvolvido na referida instituição, como importante em nossa formação. Através deste, são disponibilizados semanalmente os grupos estudos do luto (GEL), ou seja, grupos centrados na tarefa de abordar o tema da morte e do morrer, reunindo alunos da graduação, pós-graduação, Residência Multiprofissional em Saúde e outros profissionais. Além disso, somos inclinados a escutar profissionais que atuam em outras instituiçõos, bem como são ministradas palestras mensais referente ao tema, abertas à comunidade e uma das quais pudemos também realizar. No GEL, foi possível compartilhar experiências, sentimentos, percepções, condutas clínicas, entre outras, refletindo sobre o viver e o morrer.

Considerando que o hospital, conforme Angerami-Camon (2010, p. 153), "é uma instituição marcada entre a vida e a morte", acredita-se que esse espaço possibilita não somente a troca de conhecimentos entre diferentes profissionais, mas também de experiências em conviver diariamente com a morte. Ao deparar com a constatação ou iminência de morte de seu paciente, os profissionais, que, antes de tudo, são pessoas, deparam com sua impotência especialmente nos casos em que o paciente encontra-se fora de possibilidades terapêuticas curativas (FPTC).

Discutir sobre a temática da morte, portanto, coloca-se como uma atitude de essencial relevância, visto que, no hospital, a evidência de morte está tão escancarada que é praticamente impossível colocá-la de lado. Observamos que um espaço de demanda espontânea em que se possa falar do processo de morte e morrer proporciona aos profissionais de diferentes campos de saber a possibilidade de expressarem seus sentimentos, de sentirem-se humanos, de trocarem vivências cotidianas angustiantes e, consequentemente, aprender com seus pares modos de conviver com a finitude nossa de cada dia. 


\section{Considerações finais}

No que diz respeito ao ser residente, podemos afirmar que essa experiência foi um marco em nossa trajetória profissional. Aprender e intervir em conjunto, de fato, foi uma tarefa ímpar, pois, mais do que aplicar conhecimentos acadêmicos específicos, vivenciamos o desenvolvimento de habilidades pessoais e interpessoais.

Ampliamos o alcance da Psicologia como ciência e profissão quando também nos desenvolvemos como pessoa. Concluímos pela necessidade e importância do trabalho em equipe, conscientes da inviabilidade de um caminho solitário em saúde.

Posicionando-nos dessa maneira, enfatizamos nosso papel como psicólogos na instituição hospitalar, intervindo para o bem-estar integral do paciente oncológico, cientes de que a tarefa de se inserir simbolicamente em uma equipe multiprofissional é contínua e independente do tempo e do espaço em que nos encontramos. Para isso, devemos estar cientes da importância dos vínculos estabelecidos entre os profissionais e entre profissionais-usuários, assim como da necessidade de interação entre os diversos saberes visando a uma assistência integral e humanizada, de forma a atender aos princípios de universalidade, integralidade e equidade.

\section{Referências}

Angerami-Camon, V. (2010). E a psicologia entrou no hospital. São Paulo: Cengage Learning.

Angerami-Camon, V. (org.). (2002). Psicologia hospitalar: teoria e prática. São Paulo: Pioneira-Thomson Learning.

Brasil. Ministério da Saúde (2006). Residência multiprofissional em saúde: experiências, avanços e desafios. Brasília: Ministério da Saúde. Recuperado em 18 de novembro de 2011, de http://www.saude.gov.br/bvs.

Bromberg, M. H. (1998). Cuidados paliativos para o paciente com câncer: uma proposta integrativa com a equipe, paciente e famílias. In: M. M. Carvalho. Resgatando o viver: Psico-oncologia no Brasil. (pp. 186-231). São Paulo: Summus. 
Dallegrave, D. (2008). No olho do furação, na ilha da fantasia: a invenção da residência multiprofissional em saúde. Dissertação de mestrado, Faculdade de Enfermagem, Universidade Federal do Rio Grande do Sul, Porto Alegre.

Franco, M. H. (2008). Trabalho com pessoas enlutadas. In: V. A. Carvalho et al. Temas em psico-oncologia. (pp. 398-402). São Paulo: Summus.

Freud, S. (1996). O estranho. In: Sigmund Freud. Edição standard brasileira das obras completas de Sigmund Freud (Jayme Salomão, trad., Vol. 17, pp. 235269). Rio de Janeiro: Imago. (Trabalho original publicado em 1919).

Instituto Nacional do câncer. (1997). O problema do câncer no Brasil. (4. ed.) Rio de Janeiro: INCA.

Ismael, S. (2005). A inserção do psicólogo no contexto hospitalar. In: S. Ismael. (org.). A prática psicológica e sua interface com as doenças. (pp.17-36). São Paulo: Casa do Psicólogo.

Kovács, M. J. (1992). Morte e desenvolvimento humano. São Paulo: Casa do Psicólogo.

Leshan, L. (1994). Brigando pela vida: aspectos emocionais do câncer. São Paulo: Summus.

Liberato, R. P., Carvalho, V. A. (2008). Psicoterapia. In: V. A. Carvalho et al. Temas em Psico-oncologia. (pp. 341-350). São Paulo: Summus.

Moré, C., Crepaldi, M., Queiroz, A., Wendt, N. \& Cardoso, V. (2004). As representações sociais do psicólogo entre os residentes do programa de saúde da família e a importância da interdisciplinaridade. Psicologia Hospitalar, $1(1), 59-75$.

Silva, Q. T. A. (2010). Residência multiprofissional em saúde: o estar junto na formação dos residentes em saúde. Dissertação de mestrado, Faculdade de Educação, Universidade Federal do Rio Grande do Sul, Porto Alegre.

Simonetti, A. (2004). Manual da psicologia hospitalar: o mapa da doença. São Paulo: Casa do Psicólogo.

Sontag, S. (2004). Doença como metáfora: AIDS e suas metáforas. São Paulo: Companhia das Letras.

Zannon, C. (1994). Desafios à psicologia na instituição de saúde. Psicologia: Ciência e Profissão, 13, 16-21. 